\title{
Thermoplastic matrix material selection using multi criteria decision making method for hybrid polymer composites
}

\begin{abstract}
Multi criteria decision making (MDCM) methods are amongst the approaches available in aiding composite designers to make the final decision especially during the material selection process where multiple solutions are present and various requirements are required to be satisfied simultaneously. Thus, in this paper, material selection process of thermoplastic matrix using MDCM methods for hybrid natural fiber/glass fiber polymer composites is presented. The aim is to identify the most suitable type of thermoplastic matrix to be used in the hybrid polymer composites formulation. The Weighted Sum Method (WSM) is applied in the selection process of seven candidate thermoplastic matrix materials based on the product design specifications. The overall analysis highlights that low density polyethylene (LDPE) is the preferred matrix for the intended application based on the highest scores obtained compared to other candidate materials. A signal-to-noise $(\mathrm{S} / \mathrm{N})$ ratio analysis was further performed to validate the initial selection results where LDPE once again outperformed other candidate materials with highest $\mathrm{S} / \mathrm{N}$ ratio score in the non-compensatory approach.
\end{abstract}

Keyword: Hybrid polymer composites; Material selection; Multi criteria decision making;Signal-tonoise ratio (SNR); Thermoplastic matrix 